\title{
Plasticidade da aprendizagem de Diachasmimorpha longicaudata (Hymenoptera: Braconidae) associada a voláteis de frutos e óleos essenciais
}

\author{
Willian C.Zadra (1), Josué Sant’Ana (1), Luiza R. Redaelli (1) \& Roberta Tognon (D)
}

Programa de Pós-Graduação em Fitotecnia, Faculdade de Agronomia, Universidade Federal do Rio Grande do Sul, Av. Bento Gonçalves, $7712,91540-000$ Porto Alegre, RS, Brasil. (willian.zadra@ufrgs.br; josue.santana@ufrgs.br; luredael@ufrgs.br; roberta.tognon@ufrgs.br)

Recebido 9 Agosto 2017

Aceito 28 Maio 2018

Publicado 21 Junho 2018

DOI: $10.1590 / 1678-4766 e 2018026$

RESUMO. Diachasmimorpha longicaudata (Ashmead, 1905) (Hymenoptera: Braconidae) é um dos parasitoides mais utilizados em programas de controle biológico de tefritídeos no mundo. Contudo pouco se sabe sobre a capacidade de aprendizagem e memória deste braconídeo na busca pelo hospedeiro Anastrepha fraterculus (Wiedemann, 1830) (Diptera: Tephritidae). Neste estudo, avaliou-se o tempo de residência (TR) e o parasitismo de fêmeas de $D$. longicaudata oriundas de larvas de $A$. fraterculus criadas em dieta artificial, goiaba ou em manga e posteriormente, expostas aos odores destes frutos. Foi observada a aprendizagem de fêmeas de D. longicaudata condicionadas na fase adulta aos voláteis de óleo essencial de baunilha (OEB) e de laranja (OEL) e avaliadas, com os mesmos, em testes de quimiotaxia. Também registrou-se a memória deste parasitoide exposto ao OEB. Os insetos utilizados nos experimentos foram mantidos em câmaras climatizadas $\left(25 \pm 2{ }^{\circ} \mathrm{C}, 70 \pm 10 \% \mathrm{UR}\right)$ na fotofase de $14 \mathrm{~h}$ (adultos) e na escotofase (imaturos). As respostas quimiotáxicas foram registradas com olfatômetro tipo "Y" e o parasitismo (condicionamento na fase imatura), em larvas mantidas em unidades com polpa de goiaba, manga ou sem polpa (controle). Fêmeas do parasitoide criadas em larvas mantidas em dieta artificial foram expostas a OEL ou OEB por $4 \mathrm{~h}$ e a aprendizagem e memória (OEB) avaliadas em olfatômetro, a cada 24 h e por até $72 \mathrm{~h}$. Fêmeas inexperientes de D. longicaudata foram mais atraídas para os voláteis de manga e goiaba em relação ao controle. No entanto, as experientes apresentaram TR maior para os odores dos frutos nos quais se desenvolveram. Contudo, quando os odores destes frutos foram oferecidos simultaneamente, o TR foi maior para os voláteis de manga. O percentual de parasitismo de vespas inexperientes foi maior na presença das polpas e, das experientes, nas larvas que continham os odores aos quais haviam sido condicionadas. O TR de fêmeas inexperientes foi significativamente maior para a acetona do que para os odores dos óleos. Fêmeas experientes em OEB responderam mais a este odor em relação ao controle, entretanto, não houve diferença para os experientes em OEL. A memória ao odor de OEB foi mantida por até $48 \mathrm{~h}$. Concluímos que fêmeas experientes reconhecem odores aos quais se desenvolvem e aos óleos aos quais receberam experiência, resultando em preferência a estes quanto ao tempo de residência. Entretanto, reconhecer fatores que interferem na comunicação entre hospedeiro-parasitoide pode possibilitar maior adequação e confiabilidade na utilização de $D$. longicaudata em programas de controle biológico.

PALAVRAS-CHAVE. Quimiotaxia, parasitismo, mosca-das-frutas, memória.

ABSTRACT. Learning plasticity of Diachasmimorpha longicaudata (Hymenoptera: Braconidae) associated with fruit and essential oil volatiles. Diachasmimorpha longicaudata (Ashmead, 1905) (Hymenoptera: Braconidae) is one of the most used parasitoids in biological control programs of tephritids worldwide. Nevertheless, the knowledge about search strategies related to its learning and memory ability for finding its host Anastrepha fraterculus (Wiedemann, 1830) (Diptera: Tephritidae) is still limited. We observed residence time (RT) and parasitism of D. longicaudata females from A. fraterculus larvae reared on artificial diet, guava or mango and later exposed to these fruits odors. We registered the learning behavior of female parasitoids conditioned with vanilla essential oil (VEO) and orange essential oil (OEO) and evaluated with the same volatiles in chemotaxis' bioassays. We also recorded the memory of this parasitoid exposed to VEO. Insects were kept under controlled chambers $\left(25 \pm 2{ }^{\circ} \mathrm{C}, 70 \pm 10 \% \mathrm{RH}\right)$ with $14 \mathrm{~h}$ photophase (adults) or in the scotophase (immature). The chemotactic responses were recorded with a "Y" olfactometer and the parasitism (immature stage conditioning), in larvae kept in fractions with guava pulp, mango or without pulp (control). Parasitoids females reared on larvae maintained on artificial diet were exposed to VEO or OEO for $4 \mathrm{~h}$ and learning and memory (VEO) evaluated in olfactometer, every 24 until $72 \mathrm{~h}$. Inexperienced females of $D$. longicaudata were more attracted to volatiles of mango and guava when contrasted with control. Nevertheless, the experienced ones presented higher RT to the odors from fruits that they developed. However, when the odors of these fruits were offered simultaneously, the RT was higher for mango volatiles. The percentage of parasitism of inexperienced wasps was higher on the presence of pulps and, to experienced, in the larvae with odors which it had been conditioned. RT of inexperienced females was significantly higher for acetone than for odors of oils. Female with previous contact with VEO responded more to this odor than to control, however, no differences were found in chemotactic responses of females pre-exposed to OEO. VEO odor memory was maintained at least 48 hours. We conclude that experienced females recognized odors to which they developed and the oils to which they have been given experience, resulting in preference as to residence time, resulting in preference to them as to the residence time. However, recognizing interference factors in host-parasitoid communication may allow greater adequacy and reliability to use $D$. longicaudata in biological control programs.

KEYWORDS. Chemotaxy, parasitism, fruit fly, memory. 
Em sistemas tritróficos, voláteis provenientes de plantas atraem parasitoides (TurLINGS \& Ton, 2006; WyCKhuYs \& HeIMPEL, 2007; BELda \& RiUdAVETS, 2010), os quais utilizam estas pistas na localização de hospedeiros (Mattiacci et al., 1995; Blassioli Moraes et al., 2005; FAtouros et al., 2005; Tentelier et al., 2005). A forma como estes insetos interpretam a informação pode ser um comportamento inato (VET et al., 1995; VINSON, 1998) ou modificado (experiência), podendo resultar em uma aprendizagem (MATTHEws \& MATTHEws, 2010). A aprendizagem olfativa pode ser oriunda de pistas aprendidas no estágio imaturo (condicionamento pré-imaginal) e manifestadas no adulto (CORBET, 1985; TURLINGS et al., 1993) ou mesmo obtidas durante a fase adulta (TAKASU \& LEWIS, 2003). RAINS et al. (2006) observaram que o estímulo olfativo ao odor de 3-octanona, recebido na fase larval pode influenciar o comportamento quimiotáxico de adultos de Microplitis croceipes (Cresson, 1982) (Hymenoptera: Braconidae), aumentando a atratividade deste inseto ao referido composto. TAKASU \& LEWIS (2003) também haviam previamente registrado resultados similares em adultos desta mesma espécie. Segundo os autores, fêmeas alimentadas com água e sacarose, na presença de um odor (baunilha, cariofileno ou laranja), foram mais responsivas aos voláteis aos quais haviam sido condicionadas. Esta aprendizagem pode aumentar a eficiência e proporcionar uma vantagem adaptativa, reduzindo o tempo de busca pelo hospedeiro (StePhens, 1993; DuKas, 2008; CANale et al., 2014).

Diachasmimorpha longicaudata (Ashmead) (Hymenoptera: Braconidae), tem sido o agente mais utilizado em programas de controle de moscas-das-frutas (GonZÁLEZ et al., 2007; LóPEZ et al., 2009; MeIRELLES et al., 2016; VARGAS et al., 2016). Este inseto é um endoparasitoide coinobionte que oviposita no último ínstar larval de tefritídeos e completa o desenvolvimento no estágio pupal do hospedeiro (CARVALHO $\&$ NAScimento, 2002). Fêmeas de $D$. longicaudata detectam os voláteis de frutos infestados por tefritídeos (DuAN \& Messing, 2000; Carrasco et al., 2005; SiLva et al., 2007; StuHL et al., 2011). Além disso, foi constatado que mesmo na ausência de larvas de moscas-das-frutas, substratos em decomposição (SiLva et al., 2007; SEgura et al., 2012) e a presença de fungos (GREANY et al., 1977) são atrativos ao parasitoide. SALEH \& ChitTKA (2006) e Benelli et al. (2014) realizaram estudos com braconídeos e constataram mudanças de comportamento através de aprendizagem olfativa. Dentre os braconídeos, Biosteres arisanus (Sonan, 1932), Psyttalia concolor (Szépligeti, 1910) e D. longicaudata são algumas das espécies que apresentaram evidências de aprendizagem (Dukas \& Duan, 2000; Canale et al., 2014; Segura et al., 2007, 2016). Portanto, o conhecimento dos mecanismos que intermediam a comunicação química e a interação tritrófica entre $D$. longicaudata, inseto herbívoro e fruto, como a influência do hospedeiro de origem e da aprendizagem no comportamento de busca, podem resultar em práticas e produtos que favoreçam tanto o controle biológico natural quanto o aplicado. A criação em laboratório de braconídeos em larvas de moscas criadas em dieta artificial, por várias gerações, pode interferir, como já observado em outros inimigos naturais, no comportamento destes organismos na fase adulta (Bueno et al., 2014; SiqueIra et al., 2012), dificultando o encontro do parasitoide com o hospedeiro $\mathrm{e}$, consequentemente, resultando em um menor índice de parasitismo. Desta forma, a aprendizagem de $D$. longicaudata, condicionada a substâncias relacionadas ao fruto alvo, pode ser uma alternativa inovadora para manipulação comportamental destes insetos, aumentando sua performance de forrageamento a campo. Neste estudo, avaliou-se o tempo de residência (TR) e o parasitismo de fêmeas de $D$. longicaudata oriundas de larvas de $A$. fraterculus criadas em dieta artificial, goiaba ou manga e posteriormente expostas aos odores destes frutos. Foi observada a aprendizagem, em testes de quimiotaxia, de fêmeas de D. longicaudata condicionadas na fase adulta aos voláteis de óleo essencial de baunilha (OEB) e de laranja (OEL). Também foi registrado a memória deste parasitoide exposto ao OEB. A essência de baunilha foi utilizada por este odor não fazer parte do contexto ecológico do parasitoide e serviu como "odor modelo" também utilizado por outros autores (TAKASU \& LEWIS, 1996; TOGNON et al., 2013; GREGóRIO et al., 2015). O óleo de laranja foi escolhido por apresentar ação repelente para outros insetos inclusive $D$. longicaudata (bioensaios piloto), objetivando observar uma possível reversão de comportamento para este parasitoide

\section{MATERIAL E MÉTODOS}

Indivíduos de $A$. fraterculus e $D$. longicaudata foram criados segundo metodologias propostas por TERÁN (1976) e CARVALHO et al. (1998), respectivamente, no Laboratório de Biologia, Ecologia e Controle Biológico de Insetos, Departamento de Fitossanidade da Universidade Federal do Rio Grande do Sul, em câmaras climatizadas $\left(25 \pm 2{ }^{\circ} \mathrm{C}\right.$, $70 \pm 10 \%$ UR) em duas condições: fotofase de 14 horas (adultos) e escotofase (imaturos).

Criação de $\boldsymbol{A}$. fraterculus. Adultos foram acondicionados em gaiolas de madeira $(45 \times 30 \times 30 \mathrm{~cm})$, com abertura frontal tipo manga e laterais confeccionadas de tecido voile, onde recebiam água destilada e alimento, constituído de açúcar cristal, levedo de cerveja, gérmen de trigo, extrato de soja (3:1:1:1) e complexo vitamínico (Centrum Select $\left.{ }^{\circledR}\right)$, na proporção de um comprimido macerado para cada $250 \mathrm{~g}$ de dieta. Os ingredientes foram misturados e a dieta disponibilizada em placas de Petri, trocadas uma vez por semana. Após oito dias de idade, adultos de $A$. fraterculus recebiam substratos de oviposição que ficavam sobre as gaiolas conforme descrito por MeIRELLES et al. (2016). Diariamente os ovos eram retirados e colocados sobre voile azul $\left(3 \mathrm{~cm}^{2}\right)$, em placas de Petri $(9 \times 2 \mathrm{~cm})$, contendo papel filtro umedecido no fundo, acondicionadas durante $48 \mathrm{~h}$ em câmara climatizada. Após este período, colocou-se o papel filtro e o tecido com ovos, voltados para cima, sobre a dieta artificial de larvas, composta de cenoura crua descascada (125 g), cenoura cozida descascada (125 g), açúcar cristal (125 g), levedo de cerveja (25 g), água (175 mL), 
nipagin ( $1,1 \mathrm{~g})$, ácido cítrico $(3,6 \mathrm{~g})$, benzoato de sódio $(1,1 \mathrm{~g})$ e sulfato de estreptomicina $(0,5 \mathrm{~g})$. A dieta foi acondicionada em bandejas de poliestireno expandido $(18 \times 24 \mathrm{~cm})$, tampadas, enroladas com jornal e mantidas por sete dias. No sétimo dia, os pacotes foram abertos e as bandejas colocadas em recipientes plásticos $(45 \times 30 \mathrm{~cm})$, sobre uma camada de $1 \mathrm{~cm}$ de areia esterilizada, cobertas com voile, onde permaneciam por sete dias. Decorrido este período, descartou-se a areia e a dieta. Os pupários obtidos foram colocados em potes plásticos $(100 \mathrm{~mL})$ com abertura na tampa $(2 \times 2 \mathrm{~cm})$ protegida com voile, contendo areia esterilizada no fundo, permanecendo por cerca de 10 dias.

Criação de D. longicaudata. Adultos de $D$. longicaudata permaneceram em gaiolas de madeira $(45 \times 60 \times 20 \mathrm{~cm})$, coberta com voile nas laterais, recebendo água disponibilizadas em potes plásticos $(100 \mathrm{~mL}) \mathrm{com}$ tampa perfurada, contendo tiras de tecido Spontex Resist ${ }^{\circledR}$ e dieta, de consistência gelatinosa, disposta em placas de Petri composta por: água $(120 \mathrm{~mL})$, mel $(120 \mathrm{~mL})$, ágar $(0,8 \mathrm{~g})$, ácido ascórbico $(0,05 \mathrm{~g})$ e nipagin $(0,005 \mathrm{~g})$, trocada semanalmente. Larvas de terceiro instar de $A$. fraterculus (aproximadamente dez dias de idade) foram expostas por 90 minutos, em unidade de parasitismo, confeccionada com tampa de caixa Gerbox $(11 \times 11 \times 3,5 \mathrm{~cm})$, com recorte central circular de aproximadamente $10 \mathrm{~cm}$ de diâmetro, coberto com tecido voile. As larvas foram colocadas sobre este tecido, pela parte de dentro da tampa e o fundo da caixa Gerbox colocado sobre as larvas, servindo como base. A tampa e a caixa foram fixadas uma à outra com o auxílio de atilhos de borracha. Após esse período, as larvas foram colocadas em Gerbox com areia, contendo na tampa uma abertura $(9 \times 9 \mathrm{~cm})$, protegida com voile, onde permaneceram até a emergência dos parasitoides em condições controladas.

Condicionamento pré-imaginal de $\boldsymbol{D}$. longicaudata. Foram utilizados frutos de goiaba vermelha [Psidium guajava L. (Myrtales: Mirtaceae)], var. Paluma e manga [Mangifera indica L. (Sapindales: Anacardiaceae)], var. Tommy para o desenvolvimento das larvas do hospedeiro. Estes frutos foram higienizados com hipoclorito de sódio (2\%) por aproximadamente 30 minutos. Posteriormente foi feita uma abertura na casca dos frutos retirando-se cerca de $1 \mathrm{~g}$ de polpa. Por este orifício foi feita uma infestação artificial de larvas de A. fraterculus (aproximadamente 50) em cada fruto. Foram utilizadas larvas de segundo instar oriundas da criação, mantidas em dieta artificial. Os frutos foram acondicionados em potes plásticos $(500 \mathrm{~mL})$, cobertos com tecido voile, contendo areia esterilizada no fundo, onde permaneceram por aproximadamente por 4 dias (até as larvas atingirem o $3^{\circ}$ instar larval (Altafini et al., 2013). Após este período, as larvas foram retiradas dos frutos, expostas à $D$. longicaudata em unidades de parasitismo (90 min.) e após acondicionadas em Gerbox com areia, conforme descrito na criação de D. longicaudata, onde permaneceram até a emergência dos parasitoides em condições controladas. Após a emergência, fêmeas com idade entre 4-6 dias foram submetidas aos testes de quimiotaxia e parasitismo.
Condicionamento adulto de D. longicaudata. Fêmeas criadas em larvas mantidas em dieta artificial foram acondicionadas em potes plásticos $(100 \mathrm{~mL})$ contendo um papel filtro $(4 \times 4 \mathrm{~cm})$ (Whatman, $80 \mathrm{~g} / \mathrm{m} 2)$ impregnado com $40 \mu \mathrm{L}$ de óleo essencial de laranja (OEL) Bioessência ${ }^{\circledR}$ ou óleo essencial de baunilha (OEB) Dr. Oetker ${ }^{\circledR}$, nas concentrações de $1 \mathrm{~mL} / \mathrm{L}(1 \%)$ e $30 \mathrm{~mL} / \mathrm{L}(30 \%)$ em solvente (acetona), respectivamente, durante 4 horas. Estes indivíduos foram considerados experientes em OEL ou OEB e utilizados nos bioensaios logo após o condicionamento. Nos testes de memória utilizaram-se somente os insetos expostos ao OEB os quais foram submetidos a este odor após $0,24,48$ e $72 \mathrm{~h}$ da exposição. Adultos considerados inexperientes foram acondicionados na presença de um papel previamente impregnado com $40 \mu \mathrm{L}$ de acetona pelo mesmo período de tempo.

Olfatometria. Os testes foram realizados em olfatômetro tubular tipo "Y" de vidro, com $6,5 \mathrm{~cm}$ de diâmetro, $21 \mathrm{~cm}$ de arena inicial e $10 \mathrm{~cm}$ em cada braço. Os bioensaios foram conduzidos em sala climatizada durante a fotofase, sob luz fluorescente (60W, $980 \mathrm{lux}$ ). Um fluxo de ar, previamente filtrado com carvão ativo, foi conduzido para dentro do olfatômetro com o auxílio de uma bomba de ar conectada a um fluxímetro e um umidificador, a uma taxa de $0,9 \mathrm{~L} / \mathrm{min}$. Este foi invertido no sentido horizontal (rotação de $180^{\circ}$ ) a cada cinco repetições e, a cada dez, foi lavado com sabão neutro, álcool etílico e água destilada, sendo posteriormente seco em estufa de esterilização a $150{ }^{\circ} \mathrm{C}$. Após este procedimento os tratamentos foram renovados. Antes do início dos experimentos as fêmeas foram individualizadas, aclimatadas por $30 \mathrm{~min}$ na sala de testes e inseridas individualmente na arena. Foi registrado o tempo de residência (TR), em segundos, dos parasitoides em cada braço, por até $5 \mathrm{~min}$. Foram considerados não responsivos os insetos que não se movimentaram ou que não alcançaram nenhum dos dois braços do olfatômetro durante os $5 \mathrm{~min}$. Realizaram-se 30 repetições por contraste. Os tempos de residência, em todos os bioensaios de olfatometria, foram testados quanto à normalidade e submetidos à Kruskal-Wallis, com $95 \%$ de confiabilidade, utilizando o software Bioestat $5.3^{\circledR}$ (Ayres et al., 2007).

Bioensaios com insetos condicionados na fase imatura. Em cada braço do olfatômetro, foi acoplada uma câmara de vidro (29,5 cm altura e $12,5 \mathrm{~cm}$ diâmetro), na qual foi colocado um pedaço de fruto sadio $(35 \mathrm{~g})$ de goiaba ou manga. Foi avaliado o tempo de residência de fêmeas de D. longicaudata com cinco dias de idade, oriundas de larvas provenientes de dieta artificial, goiaba ou manga. Foram contrastados os odores dos frutos com ar e entre eles.

Bioensaios com insetos condicionados na fase adulta. Fêmeas criadas em larvas mantidas em dieta artificial foram acondicionadas em potes plásticos $(100 \mathrm{~mL})$ contendo um papel filtro $(4 \times 4 \mathrm{~cm})$ (Whatman, $\left.80 \mathrm{~g} / \mathrm{m}^{2}\right)$ impregnado com $40 \mu \mathrm{L}$ de óleo essencial de laranja (OEL) Bioessência $^{\circledR}$ ou óleo essencial de baunilha (OEB) Dr. Oetker $^{\circledR}$, nas concentrações de $1 \mathrm{~mL} / \mathrm{L}(1 \%)$ e $30 \mathrm{~mL} / \mathrm{L}$ 
(30\%) em solvente (acetona), respectivamente, durante quatro horas. Estes indivíduos foram considerados experientes em OEL ou OEB e utilizados nos bioensaios logo após o condicionamento. Nos testes de memória utilizaram-se somente os insetos expostos ao $\mathrm{OEB}$ os quais foram avaliados submetendo-os a este odor após $0,24,48$ e 72 h da exposição. Adultos considerados inexperientes foram acondicionados na presença de um papel previamente impregnado com 40 $\mu \mathrm{L}$ de acetona pelo mesmo período de tempo. As respostas quimiotáxicas ao condicionamento com os óleos foram avaliadas inserindo na extremidade de um dos braços do olfatômetro um papel filtro $(4 \times 15 \mathrm{~cm})$ (Whatman, $80 \mathrm{~g} /$ $\mathrm{m}^{2}$ ), dobrado em forma de gaita, contendo $10 \mu \mathrm{L}$ de cada extrato (OEL ou OEB) e, na outra, o mesmo volume do solvente acetona. Todos os papeis filtro foram mantidos em câmara de fluxo por 5 min, para evaporação da acetona, antes do uso. Foram contrastados os odores dos óleos com o solvente e entre eles.

Parasitismo. Quatro fêmeas e dois machos de $D$. longicaudata (5 a 10 dias de idade), oriundos de larvas mantidas em dieta artificial, goiaba ou manga foram expostos, em gaiolas de madeira $(45 \times 30 \times 30 \mathrm{~cm})$, a escolha entre duas unidades de parasitismo, cada uma com 20 larvas de A. fraterculus de $3^{\circ}$ ínstar, oriundas de dieta artificial. Foram retiradas polpas de frutos de cada uma das espécies, as quais foram maceradas, separadamente, com auxílio de um pistilo e cadinho. Para realização dos experimentos foram contrastadas unidades pinceladas (pincel $\mathrm{n}^{\circ} 10$ ) com polpa in natura de manga ou goiaba $(2 \mathrm{~g})$ sobre o tecido voile presente na unidade de parasitismo. Unidades controle foram pinceladas apenas com água destilada. O período de exposição foi de $90 \mathrm{~min}$, após as larvas foram isoladas em caixas Gerbox ${ }^{\circledR}$ com areia e mantidas em câmara climatizada até a emergência dos parasitoides ou das moscas. Os pupários nos quais não foi observada a emergência, foram dissecados e contabilizados. Realizaram-se 10 repetições/tratamento. O percentual de larvas parasitadas e de parasitoides emergidos foi analisado por Qui-quadrado, com 95\% de confiabilidade, utilizando o software Bioestat 5.3 ${ }^{\circledR}$ (Ayres et al., 2007).

\section{RESULTADOS}

Bioensaios com insetos condicionados na fase imatura. O tempo de residência (TR) de fêmeas inexperientes (criadas em dieta artificial) de D. longicaudata foi significativamente maior tanto para os odores de goiaba $(\mathrm{H}=6,33 ; \mathrm{gl}=1 ; \mathrm{P}=0,0119)$ quanto para os de manga $(\mathrm{H}=7,6 ; \mathrm{gl}=1 ; \mathrm{P}=0,0058)$ em relação ao controle (ar). Quando os insetos foram submetidos ao contraste entre os voláteis de ambos os frutos, o TR das fêmeas foi maior para os voláteis de manga $(\mathrm{H}=10,54 ; \mathrm{gl}=1 ; \mathrm{P}=0,0012)$ (Fig. 1). Fêmeas oriundas de larvas que se desenvolveram em goiaba e manga apresentaram tempos de residência maiores para insetos provenientes de cada um destes frutos, quando comparados ao controle $(\mathrm{H}=15,68 ; \mathrm{gl}=1 ; \mathrm{P}<0,0001 \mathrm{e}$ $\mathrm{H}=28,81 ; \mathrm{gl}=1 ; \mathrm{P}<0,0001$, respectivamente). No entanto, independentemente da origem dos parasitoides, não foram observadas diferenças significativas quando os mesmos foram submetidos ao contraste entre os odores dos dois frutos (Fig. 2).

Bioensaios com insetos condicionados na fase adulta. O TR de fêmeas inexperientes de $D$. longicaudata foi significativamente maior para a acetona do que para os odores dos óleos de laranja (OEL) $(\mathrm{H}=9,98 ; \mathrm{gl}=1 ; \mathrm{P}=0,0016)$

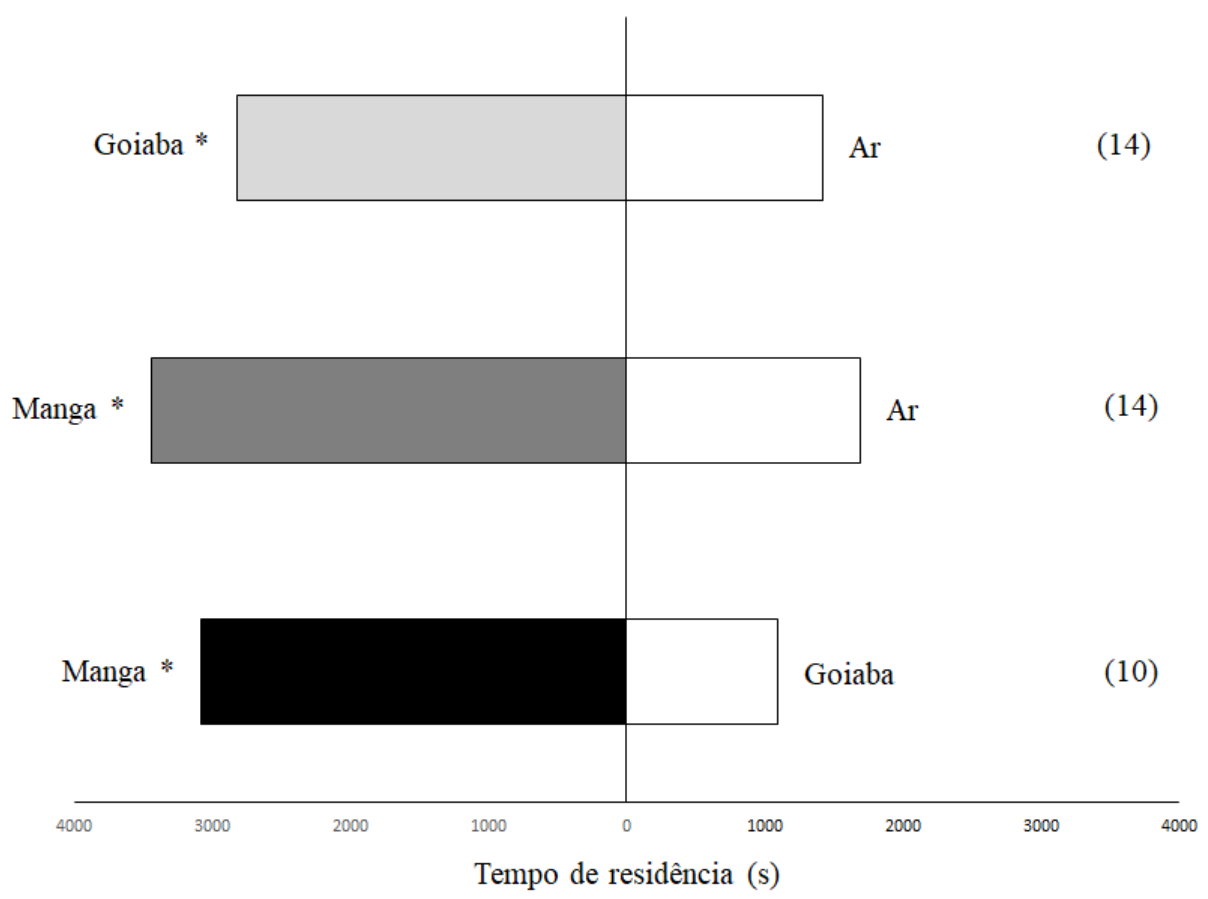

Fig.1. Tempo de residência (s) de fêmeas de Diachasmimorpha longicaudata (Ashmead, 1905) oriundas de larvas de Anastrepha fraterculus (Wiedemann, 1830) criadas em dieta artificial (inexperientes) submetidas ao ar (controle) e aos odores de goiaba ou manga. Tempo de observação: 5 min. Número de insetos não responsivos entre parênteses. * Diferença significativa pelo teste de Kruskal-Wallis $(\mathrm{P}<0,05)$. 
e baunilha (OEB) $(\mathrm{H}=6,65 ; \mathrm{gl}=1 ; \mathrm{P}=0,0099)$ (Fig. 3A). Fêmeas experientes em OEB responderam mais ao odor deste em relação ao controle $(\mathrm{H}=7,16 ; \mathrm{gl}=1 ; \mathrm{P}=0,0074)$ (Fig. 3B), no entanto não houve diferença para os experientes em OEL ( $\mathrm{P}>0,05)$ (Fig. 3C). Fêmeas de D. longicaudata expostas ao OEB mantiveram memória a este odor por até
24 horas. O comportamento inato foi novamente evidenciado em 48 horas $(\mathrm{H}=10,69 ; \mathrm{gl}=1 ; \mathrm{P}<0,05)$ (Fig. 4).

Parasitismo. Fêmeas de $D$. longicaudata provenientes de larvas de $A$. fraterculus criadas em dieta artificial (inexperientes) parasitaram um percentual maior de larvas na presença da polpa de goiaba $(34 \%)\left(\chi^{2}=4,94 ; \mathrm{gl}=1 ; \mathrm{P}=0,0364\right)$

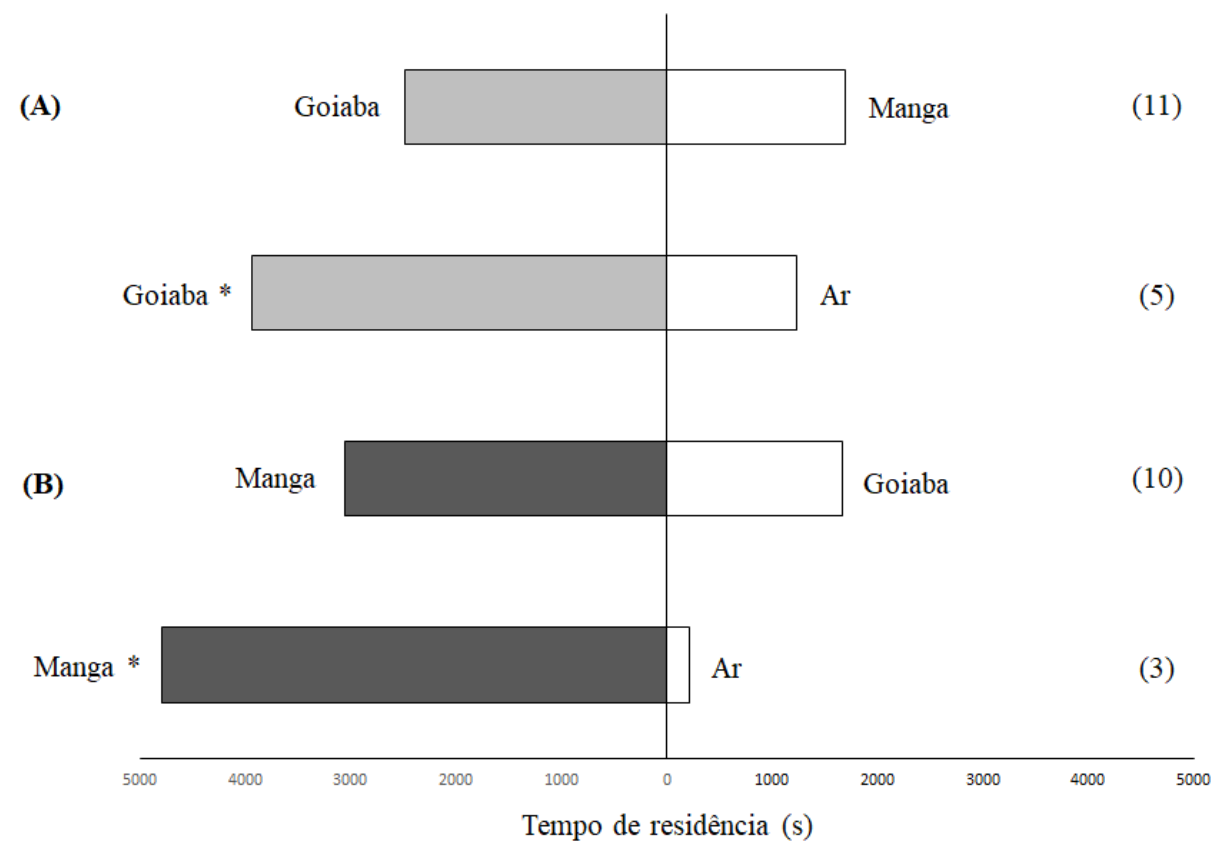

Fig. 2. Tempo de residência (s) de fêmeas de Diachasmimorpha longicaudata (Ashmead, 1905) oriundas de larvas de Anastrepha fraterculus (Wiedemann, 1830) criadas em goiaba (experientes em goiaba) (A) ou em manga (experientes em manga) (B), aos contrastes de: ar (controle), odores de pedaços de frutos in natura de goiaba ou manga. Tempo de observação: 5 min. Número de insetos não responsivos entre parênteses. * Diferença significativa pelo teste de Kruskal-Wallis $(\mathrm{P}<0,05)$.

(A)

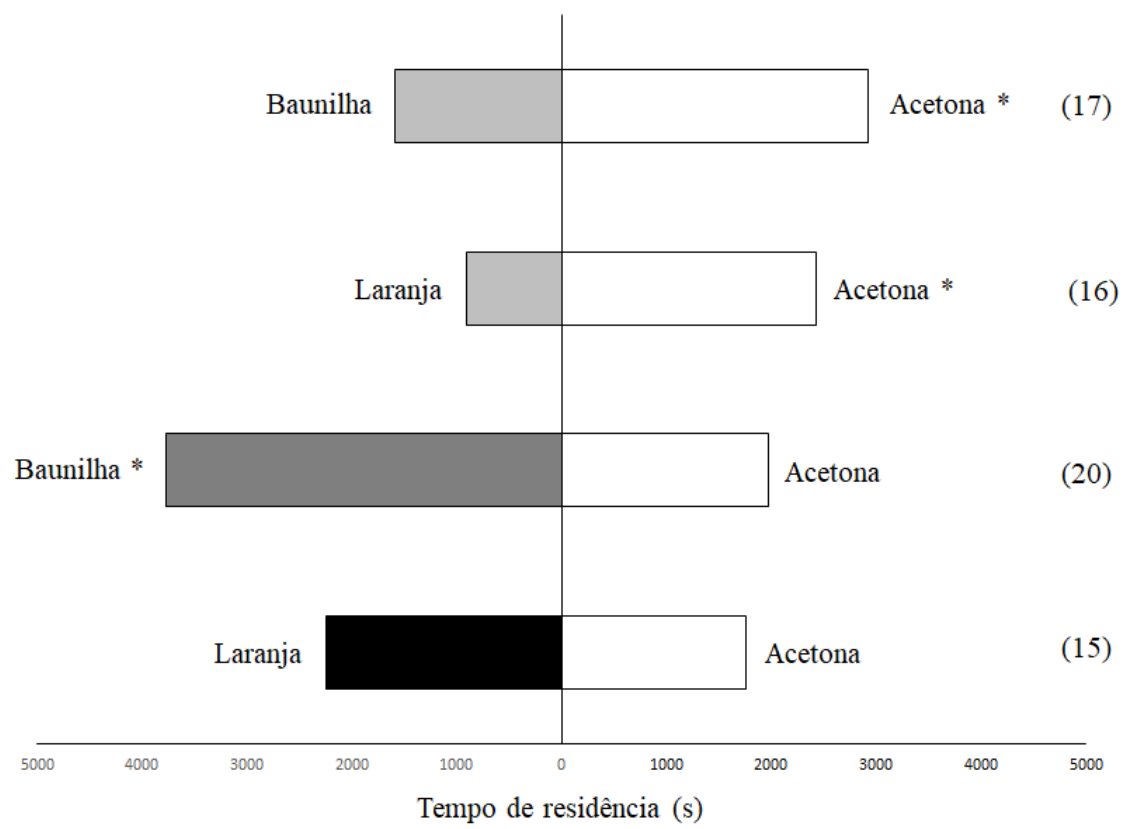

Fig. 3. Tempo de residência (s) de fêmeas de Diachasmimorpha longicaudata (Ashmead, 1905) oriundas de larvas de Anastrepha fraterculus (Wiedemann, 1830) criadas em dieta artificial exposta à acetona (inexperientes) (A); experientes ao óleo essencial de baunilha (OEB) (B) e experientes ao óleo essencial de laranja (OEL) (C), submetidas ao contraste entre ar e os óleos. Tempo de observação: 5 min. Número de insetos não responsivos entre parênteses. *Diferença significativa pelo teste de Kruskal-Wallis $(\mathrm{P}<0,05)$. 


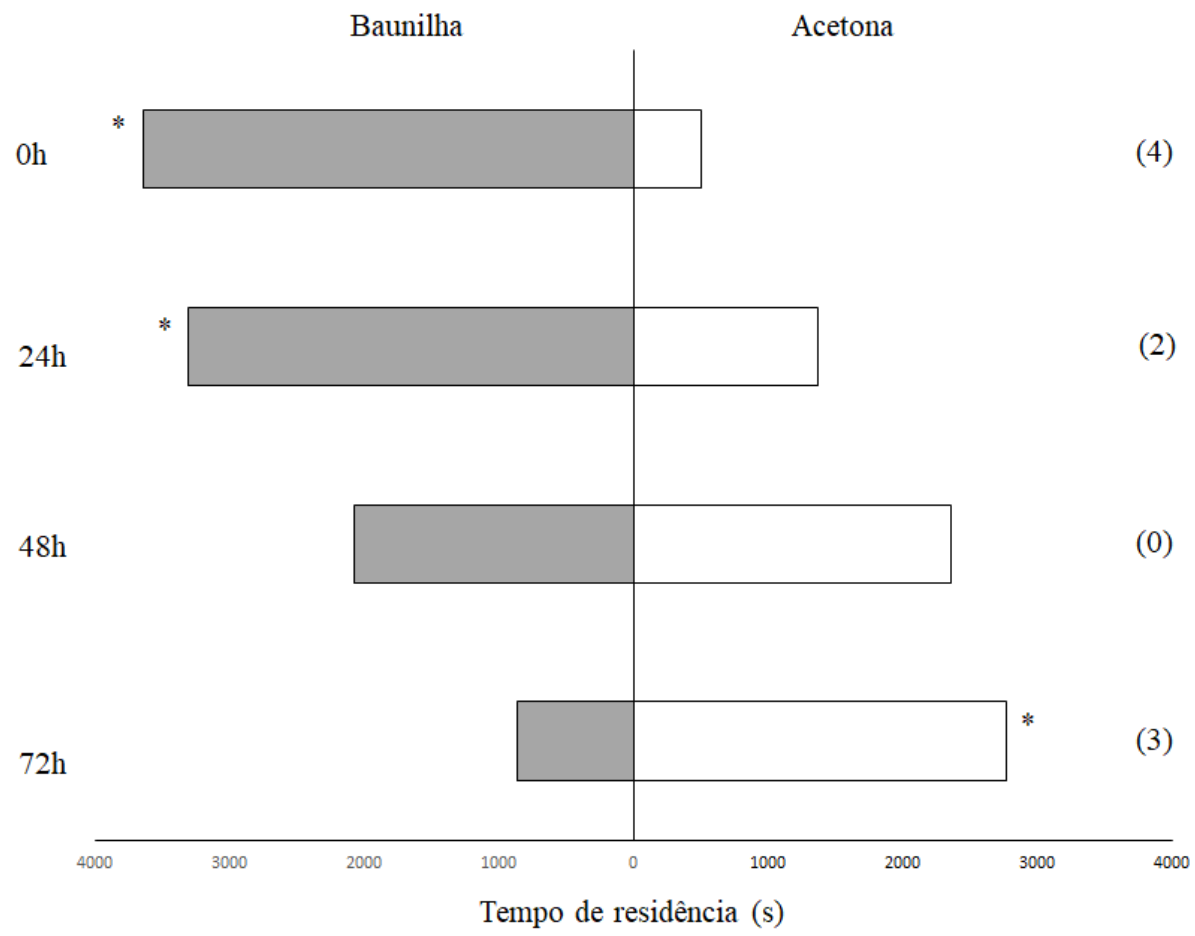

Fig. 4. Tempo de residência (s) de fêmeas de Diachasmimorpha longicaudata (Ashmead, 1905) que estiveram em contato com óleo essencial de baunilha (OEB) (experientes em baunilha), submetidas aos contrastes de acetona (controle) e odor de baunilha. Tempo decorrido após experiência com OEB: 0 , 24, 48 e 72 horas. Tempo de observação: 5 min. Número de insetos não responsivos entre parênteses. *Diferença significativa pelo teste de KruskalWallis $(\mathrm{P}<0,05)$.

e manga $(25 \%)\left(\chi^{2}=8,1 ; \mathrm{gl}=1 ; \mathrm{P}=0,0072\right)$, quando ambos os tratamentos foram contrastados com o controle (larvas sem polpa). Larvas com a presença da polpa de goiaba foram mais parasitadas $(21 \%)$ em relação às que continham manga $(13 \%)$ $\left(\chi^{2}=7,52 ; \mathrm{gl}=1 ; \mathrm{P}=0,0101\right)$, em exposição simultânea. $\mathrm{O}$ sucesso de parasitismo diferiu apenas na presença de polpa de goiaba $(23 \%)$ em contraste com controle $(8,5 \%)\left(\chi^{2}=26,69\right.$; $\mathrm{gl}=1 ; \mathrm{P}<0,0001)$. Parasitoides provenientes de larvas criadas em goiaba (experientes em goiaba) parasitaram mais larvas na presença deste odor $(49 \%)\left(\chi^{2}=36,38 ; \mathrm{gl}=1\right.$; $\mathrm{P}<0,0001$ ), além disso, o sucesso de parasitismo também foi maior neste tratamento (28\%), em comparação com o controle $(14,5 \%)\left(\chi^{2}=17,15 ; \mathrm{gl}=1 ; \mathrm{P}<0,0001\right)$. De forma semelhante, fêmeas oriundas de larvas criadas em manga (experientes em manga) parasitaram mais insetos na presença da polpa deste fruto $(32 \%)$, diferindo do controle $(15 \%)$ $\left(\chi^{2}=12,29 ; \mathrm{gl}=1 ; \mathrm{P}<0,001\right)$, entretanto não houve diferença no sucesso de parasitismo $\left(\chi^{2}=3,12 ; \mathrm{gl}=1 ; \mathrm{P}=0,1116\right)$. Quando foram contrastadas as respostas frente aos odores de manga e goiaba, o parasitismo $(38,5 \%)$ e o sucesso (33\%) foram superiores em manga $\left(\chi^{2}=31,5 ; \mathrm{gl}=1 ; \mathrm{P}<0,0001\right.$; $\chi^{2}=46,55 ; \mathrm{gl}=1 ; \mathrm{P}<0,0001$ respectivamente).

\section{DISCUSSÃO}

Os resultados obtidos a partir dos testes de olfatometria e parasitismo mostraram que as fêmeas inexperientes de D. longicaudata são atraídas por voláteis oriundos de manga e goiaba, corroborando o observado por EBEN et al. (2000), os quais constataram que compostos liberados por frutos sadios e infestados de plantas hospedeiras atuam nos mecanismos de localização e busca deste parasitoide. Ainda em relação à atratividade, CARRASCO et al. (2005) verificaram que D. longicaudata foi mais responsiva a odores de manga infestada por Anastrepha spp. ou com danos mecânicos do que aos de frutos sadios.

As respostas positivas de fêmeas inexperientes aos odores de manga e goiaba quando contrastadas com o ar, estão provavelmente associadas à plasticidade deste parasitoide em relação à percepção de voláteis de diferentes frutos, sendo esta uma estratégia evolutiva para atraí-los a habitats onde encontram-se os sítios de oviposição. O reconhecimento do odor de diferentes frutos pelo parasitoide está provavelmente relacionado à $D$. longicaudata parasitar moscas em diferentes hospedeiros (MonTOYA et al., 2000).

Além de perceber voláteis oriundos de diferentes frutos, $D$. longicaudata foi capaz de discriminá-los em exposições simultâneas, tanto em testes de parasitismo como nos de quimiotaxia. Este resultado evidencia que existe uma preferência inata da espécie por odores provenientes de manga. No entanto, após a experiência em frutos de goiaba este comportamento muda e o inseto não mais diferencia os odores destes dois frutos, possivelmente, porque passou a reconhecer também voláteis de goiaba, não os distinguindo quando expostos de forma conjunta. LeYva et al. (1991) já haviam observado que fêmeas de $D$. longicaudata foram significativamente mais atraídas a voláteis de citros em comparação com os de pêssego e manga. Um padrão 
hierárquico de preferência aos voláteis dos frutos figo, pêssego, laranja e maçã, respectivamente, foi constatado por Segura et al. (2016) em fêmeas desta espécie. No nosso estudo observou-se também um padrão no comportamento quimiotáxico com fêmeas inexperientes preferindo odores de manga. No entanto, o padrão pode variar em função dos tipos de receptores envolvidos, olfativos (antenas) e gustativos (aparelho bucal e ovipositor) (ATKINs, 1980), uma vez que no parasitismo a maior preferência observada no nosso estudo foi para goiaba.

Apesar dos adultos apresentarem um comportamento inato em relação aos odores de frutos, verificou-se que é possível manipular o mesmo, ou seja, parasitoides que se desenvolveram em larvas oriundas de manga e/ou goiaba modificaram seu direcionamento quimiotáxico e os percentuais de parasitismo em comparação as inexperientes, evidenciando um processo de aprendizagem. A experiência desempenha um papel importante na biologia dos insetos, uma vez que a aquisição de aprendizagem na fase larval pode permitir que, na idade adulta, os indivíduos consigam localizar seus hospedeiros com maior facilidade. Os resultados obtidos estão relacionados ao condicionamento pré-imaginal explicado pela Hipótese do Legado Químico, a qual prediz que compostos químicos presentes no ambiente de desenvolvimento larval, na hemolinfa ou na camada externa do hospedeiro, tem influência sobre a escolha hospedeira do parasitoide adulto (CORBET, 1985). Esta hipótese foi posteriormente confirmada por estudos realizados com parasitoides (STORECK et al., 2000). Os resultados do nosso trabalho evidenciaram que houve modificação na percepção química de $D$. longicaudata, provavelmente, relacionada a este legado. De forma semelhante, SMITH \& CoRnell (1979) observaram que houve mudança no comportamento de busca e, consequentemente, aprendizado aos odores do hospedeiro, potencializando o encontro das fêmeas de Nasonia vitripennis (Walker, 1836) (Hymenoptera: Pteromalidae), assim como o observado em D. longicaudata. VillagRa et al. (2007) já haviam relatado que experiências adquiridas em fases larvais afetam as respostas em adultos de Aphidius ervi Haliday, 1834 (Hymenoptera: Braconidae) para o encontro de seu hospedeiro. As modificações na percepção após a experiência podem estar associadas à síntese de proteínas de ligação (odor binding proteins, $O B P S$ ) específicas presentes nas antenas dos insetos capazes de reconhecer determinadas moléculas, desempenhando um papel importante na percepção e discriminação de diferentes odores (ZHOU, 2010; ZHANG et al., 2011). Quando o inseto se desenvolve em dieta não há contato com odores de frutos, no entanto, quando criado nestes, pode haver formação de $O B P S$ capazes de detectar diferentes voláteis. Respostas quimiotáxicas semelhantes de fêmeas experientes em goiaba ou em manga, estão possivelmente relacionadas ao fato de que estes frutos compartilham vários compostos (PINO et al., 2005; CHEN et al., 2006), sendo assim o inseto passou a identificá-los e não mais distingui-los, quando expostos de forma conjunta.
A aprendizagem em $D$. longicaudata também pode ocorrer na fase adulta. Este fato está relacionado ao princípio de Hopkins, o qual enfatiza a importância da experiência na fase adulta na escolha do hospedeiro (BARRON, 2001). Em nossos resultados foi possível observar que a exposição de fêmeas de $D$. longicaudata a odores não relacionados à história de vida deste inseto, como os óleos essenciais de baunilha e laranja, induziu uma resposta positiva de busca ao OEB e indiferente (não significativa) ao OEL, mesmo sendo este último repelente para muitos insetos (REGNAULT-RoGER et al., 2012). Desta forma, o parasitoide provavelmente passou a associar o odor de baunilha à presença de um possível hospedeiro. TAKASU \& LEWIS (1996) observaram que fêmeas de $M$. croceipes que permaneceram por 30 segundos na presença de um odor (baunilha ou laranja) com alimento, responderam mais a estes odores, do que as sem condicionamento. De forma semelhante VARGAS et al. (2017) verificaram que fêmeas de Trichogramma pretiosum Riley, 1879 (Hymenoptera: Trichogrammatidae) experientes ao extrato de ovos de Spodoptera frugiperda (Smith, 1979) (Lepidoptera: Noctuidae) foram mais atraídas a este odor do que ao hexano, enquanto as inexperientes responderam de forma igual aos dois tratamentos. Outras formas de aprendizado, como a visual, foram observadas por SEGURA et al. (2007), onde fêmeas de D. longicaudata treinadas em unidades de oviposição de cores diferentes (amarelo, vermelho e verde), contendo larvas do hospedeiro, visitaram e permaneceram por mais tempo nas unidades de cores em que haviam sido condicionadas. Com esta mesma espécie, em ensaios de olfatometria, foi observado que a resposta de fêmeas foi maior aos voláteis dos frutos aos quais tiveram experiência (SEgura et al., 2016).

Nós constatamos que fêmeas de $D$. longicaudata foram capazes de reter o aprendizado ao odor de OEB por até 24 horas. A persistência da aprendizagem, ou seja, a memória em insetos parasitoides já foi descrita em outras espécies. WARDLE \& BORDEN (1985) também observaram a memória em adultos de Exeristes roborator (Fabricius, 1793) (Hymenoptera: Ichneumonidae) condicionados na presença do hospedeiro [Galleria mellonella (L., 1758) (Lepidoptera: Pyralidae)]. Os autores constataram que logo após a emergência, os parasitoides apresentaram maior capacidade de oviposição em comparação com adultos condicionados após o segundo até 10 dias da emergência.

A experiência pode ser uma importante ferramenta para aprimorar a performance de parasitoides a serem liberados em programas de controle biológico. GiUNTI et al. (2015) ressaltaram que incluir treinamento associativo com estímulos visuais e olfativos em protocolos de criação em massa pode aumentar a eficiência de parasitoides. Os resultados obtidos indicam que é possível fazer com que D. longicaudata aprenda a reconhecer e memorizar voláteis durante as fases imatura e adulta. Identificar fatores que interferem na comunicação entre hospedeiro-parasitoide pode possibilitar maior adequação e confiabilidade na utilização de $D$. longicaudata em programas de controle biológico. 
Agradecimentos. Ao Conselho Nacional de Desenvolvimento Científico e Tecnológico (CNPq) pelo apoio financeiro (449738/2014-0) e bolsas concedidas a JS (306474/2015-8) e LRR (308113/2016-0).

\section{REFERÊNCIAS BIBLIOGRÁFICAS}

AltafinI, D. L.; RedAeLLI, L. R. \& JAHNKE, S. M. 2013. Superparasitism of Ceratitis capitata and Anastrepha fraterculus (Diptera: Tephritidae) by Diachasmimorpha longicaudata (Hymenoptera: Braconidae). Florida Entomologist 96:391-395.

ATKINS, M. D. 1980. Introduction to insect behavior. New York, Macmillan. 237p.

Ayres, M.; Ayres, MJr.; Ayres, D.L. \& Santos, A.A.S. 2007. BioEstat 5.0 aplicações estatísticas nas áreas da ciências bio-médicas. Belém, Brasil.

Barron, A. B. 2001. The Life and Death of Hopkins' Host-Selection Principle. Journal of Insect Behavior 14(6):725-737.

BeLDA, C. \& RiudaVETS, J. 2010. Attraction of the parasitoid Anisopteromalus calandrae (Howard) (Hymenoptera: Pteromalidae) to odors from grain and stored product pests in a Y-tube olfactometer. Biological Control 54:29-34.

Benelli, G.; Stefanini, C.; Giunti, G.; Geri, S.; Messing, R. H. \& Canale, A. 2014. Associative learning for danger avoidance nullifies innate positive chemotaxis to host olfactory stimuli in a parasitic wasp. Naturwissenschaften 101:753-757.

Blassioli Moraes, M. C.; Laumann, R.; Sujit, E. R.; Pires, C. \& Borges, M. 2005. Induced volatiles in soybean and pigeon pea plants artificially infested with the neotropical brown stink bug, Euschistus heros, and their effect on the egg parasitoid, Telenomus podisi. Entomologia Experimentalis et Applicata 115:227-237.

Bueno, R. C. O. F.; Bueno, A. F.; Xavier, M. F. C. \& Carvalho, M. M. 2014. Telenomus remus (Hymenoptera: Platygastridae) parasitism on eggs of Anticarsia gemmatalis (Lepidoptera: Eribidae) compared with its natural host Spodoptera frugiperda (Lepidoptera: Noctuidae). Annals of the Entomological Society of America 107:799-808.

Canale, A.; Geri, S. \& Benelli, G. 2014. Associative learning for hostinduced fruit volatiles in Psyttalia concolor (Hymenoptera: Braconidae), a koinobiont parasitoid of tephritid flies. Bulletin of Entomological Research 104:774-780.

Carrasco, M.; Montoya, P.; Cruz-López, L. \& Rojas, J. C. 2005. Response of the fruit fly parasitoid Diachasmimorpha longicaudata (Hymenoptera: Braconidae) to mango fruit volatiles. Environmental Entomology 34:576-583.

Carvalho, R. D. S. \& Nascimento, A. S. 2002. Criação e utilização de Diachasmimorpha longicaudata para controle biológico de moscasdas-frutas. In: PARRA, J. R. P. eds. Controle biológico no Brasil: parasitoides e predadores. São Paulo, Manole, p.65-179.

Carvalho, R. S.; Nascimento, A. S. \& Matrangolo, W. J. R. 1998. Metodologia de criação do parasitóide exótico Diachasmimorpha longicaudata (Hymenoptera, Braconidae), visando estudos em laboratório e em campo. Cruz das Almas, Embrapa, CNPMF. 16p.

Chen, H. C.; Sheu, M. J. \& Wu, C. M. 2006. Characterization of volatiles in Guava (Psidium guajava L. cv. Chung-Shan-Yueh-Pa) fruit from Taiwan. Journal of Food and Drug Analysis 14:398-402.

Corbet, S. A. 1985. Insect chemosensory responses: A chemical legacy hypothesis. Ecological Entomology 10:143-153.

Duan, J. J. \& Messing, R. H. 2000. Effects of host substrate and vibration cues on ovipositor-probing behavior in two larval parasitoids of tephritid fruit flies. Journal of Insect Behavior 13:175-186.

DuKas, R. 2008. Evolutionary biology of insect learning. Annual Review of Entomology 53:145-160.

Dukas, R. \& DuAn, J. J. 2000. Potential fitness consequences of associative learning in a parasitoid wasp. Behavioral Ecology 11:536-543.

Eben, A.; Benrey, B.; Sivinski, J. \& Aluja, M. 2000. Host species and host plant effects on performance of Diachasmimorpha longicaudata (Hymenoptera: Braconidae). Environmental Entomology 29:87-94.

Fatouros, N. E.; Bukovinszkine'Kiss, G.; Kalkers, L. A.; Gamborena, R. S.; Dicke, M. \& Hilker, M. 2005. Oviposition-induced plant cues: do they arrest Trichogramma wasps during host location? Entomologia Experimentalis et Applicata 115:207-215.
Giunti, G.; Canalle, A.; Messing, R. H.; Donati, E.; Stefanini, C.; Michaud, J. P. \& Benelli, G. 2015. Parasitoid learning: Current knowledge and implications for Biological Control. Biological Control 90:208-219.

González, P. I.; Montoya, P.; Pérez-Lachaud, G.; Cancino, J. \& Liedo, P. 2007. Superparasitism in mass reared Diachasmimorpha longicaudata (Ashmead) (Hymenoptera: Braconidae), a parasitoid of fruit flies (Diptera: Tephritidae). Biological Control 40:320-326.

Greany, P. D.; Tumlinson, J. H.; Chambers, D. L. \& Boush, G. M. 1977. Chemically mediated host finding by Biosteres (Opius) longicaudatus, a parasitoid of tephritid fruit fly larvae. Journal of Chemical Ecology 3:189-195.

Gregório, P. L. F.; SANT'AnA, J. \& Pires, P. D. 2015. Behavioral analysis of learning and memory in Anastrepha fraterculus. Entomologia Experimentalis et Applicata. 154:146-153.

Leyva, J. L.; Browning, H. W. \& Gilstrap, F. E. 1991. Effect of Host Fruit Species, Size, and Color on Parasitization of Anastrepha ludens (Diptera: Tephritidae) by Diachasmimorpha longicaudata (Hymenoptera: Braconidae). Environmental Entomology 20:1469-1474.

LóPez, O. P.; Hénault, Y.; CANCINO, J.; LAMBin, M.; CruZ-LóPEz, L. \& RoJAs, J. C. 2009. Is host size an indicator of quality in the mass-reared parasitoid Diachasmimorpha longicaudata (Hymenoptera: Braconidae)? Florida Entomologist 92:441-449.

Matthews, R. W. \& Matthews, J. R. 2010. Insect Behavior. New York, John Wiley \& Sons. 514p.

Mattiacci, L.; Dicke, M. \& Posthumus, M. A. 1995. $\beta$-Glucosidase: an elicitor of herbivore induced plant odor that attracts host-searching parasitic wasps. Proceedings of the National Academy of Sciences 92:2036-2040.

Meirelles, R. N.; Redaelli, L. R.; JahnKe, S. M.; Ourique, C. B. \& Ozorio, D. V. B. 2016. Parasitism of fruit flies (Tephritidae) in field, after the releases of Diachasmimorpha longicaudata (Ashmead) (Hymenoptera: Braconidae) in Rio Grande do Sul. Revista Brasileira de Fruticultura, 38(2):673-683.

Montoya, P.; Liedo, P.; Benrey, B.; Cancino, J.; Barrera, J. F.; Sivinski, J. \& Aluja, M. 2000. Biological Control of Anastrepha spp. (Diptera: Tephritidae) in Mango Orchards through Augmentative Releases of Diachasmimorpha longicaudata (Ashmead) (Hymenoptera: Braconidae). Biological Control 18:216-224.

Pino, J. A.; Mesa, J.; Munhoz, Y.; Martí, M. P. \& Marbot, R. 2005. Volatile components from Mango (Mangifera indica L.) cultivars. Journal of Agricultural and Food Chemistry 53:2213-2223.

Rains, G. C.; UtLey, S. L. \& Lewis, W. J. 2006. Behavioral monitoring of trained insects for chemical detection. Biotechnology Progress 22(1):2-8.

Regnault-Roger, C.; Vincent, C. \& Arnason, J. T. 2012. Essential Oils in Insect Control: Low-Risk Products in a High-Stakes World. Annual Review of Entomology 57:405-424.

Saleh, N. \& ChitTKA, L. 2006. The importance of experience in the interpretation of conspecific chemical signals. Behavioral Ecology and Sociobiology 61:215-220.

Segura, D. F.; Nussenbaum, A. L.; Viscarret, M. M.; Devescovi, F.; Bachmann, G. E.; Corley, J. C.; Ovruski, S. M. \& Cladera, J. L. 2016. Innate host habitat preference in the parasitoid Diachasmimorpha longicaudata: Functional significance and modifications through learning. PLoS ONE 11(3):1-18.

Segura, D. F.; Viscarret, M. M.; Ovruski, S. M. \& Cladera, J. L. 2012. Response of the fruit fly parasitoid Diachasmimorpha longicaudata to host and host-habitat volatile cues. Entomologia Experimentalis et Applicata 143:164-176.

Segura, D. F.; Viscarret, M. M.; Paladino, L. Z. C.; Ovruski, S. M. \& Cladera, J. L. 2007. Role of visual information and learning in habitat selection by a generalist parasitoid foraging for concealed hosts. Animal Behaviour 74:131-142.

SiLva, J. W. P.; BeNto, J. M. S. \& ZuCCHI, R. A. 2007. Olfactory response of three parasitoid species (Hymenoptera: Braconidae) to volatiles of guavas infested or not with fruit fly larvae (Diptera: Tephritidae). Biological Control 41:304-311.

Siqueira, J. R.; Bueno, R. C. O. F.; Bueno, A. F. \& Vieira, S. V. 2012. Preferência hospedeira do parasitoide de ovos Trichogramma pretiosum. Ciência Rural 42:1-5. 
Smith, M. A. \& Cornell, H. V. 1979. Hopkins host-selection in Nasonia vitripennis and its implications for sympatric speciation. Animal Behavior 27(2):365-370.

StePHens, D. W. 1993. Learning and behavioral ecology: incomplete information and environmental predictability. In: PAPAJ, R. D. \& LEWIS, A. C. eds. Insect Learning: ecological and evolutionary perspectives. New York, Chapman and Hall, p.195-218.

Storeck, A.; Poppy, G. M.; van Emden, H. F. \& Powell, W. 2000. The role of plant chemical cues in determining host preference in thegeneralist aphid parasitoid Aphidius colemani. Entomologia Experimentalis et Applicata 97:41-46.

Stuhl, C.; Sivinski, J.; Teal, P.; Paranhos, B. \& Aluja, M. 2011. Larvae promotes oviposition behavior by the biological control agent Diachasmimorpha longicaudata (Hymenoptera: Braconidae). Environmental Entomology 40:727-736.

TAKASU, K. \& LEWIS, W. J. 2003. Learning of host searching cues by the larval parasitoid Microplitis croceipes. Entomologia Experimentalis et Applicata 108:77-86.

TAKAsU, K. \& Lewis, W. J. 1996. The role of learning in adult food location by the larval parasitoid, Microplitis croceipes (Hymenoptera: Braconidae). Journal of Insect Behavior 9(2):265-281.

Tentelier, C.; Wajnberg, E. \& Fauvergue, X. 2005. Parasitoids use herbivore-induced information to adapt patch exploitation behaviour. Ecological Entomology 30:739-744.

TerÁN, H. R. 1976. Comportamiento alimentario y su correlación a la reproducción en hembras de Ceratitis capitata (Wied.) (Diptera: Tephritidae). Revista Agronómica del Noroeste Argentino 14:17-34.

TognON, R.; SANT'ANA, J.; JAHNKE, S.M. 2013. Aprendizagem e memória de Telenomus podisi (Hymenoptera, Platygastridae). Iheringia, Série Zoologia 103:266-271.

TuRlings, T. C. J. \& Ton, J. 2006. Exploiting scents of distress: the prospect of manipulating herbivore-induced plant odors to enhance the control of agricultural pests. Current Opinion in Plant Biology 9:421-427.

Turlings, T. C. J.; Wackers, F. L.; Vet, L. E. M.; Lewis, W. J. \& Tumlinson, J. H. 1993. Learning of host-finding cues by Hymenopterous parasitoids.
In: PAPAJ, D. R. \& LEWIS, A. C. eds. Insect Learning: Ecological and Evolutionary Perspectives. New York, Chapman and Hall, p.51-78. Vargas, C. C.; Redaelli, L. R.; Sant'Ana, J.; Morais, R. M. \& Padilha. P. 2017. Influência da idade do hospedeiro e da aprendizagem no comportamento quimiotáxico e no parasitismo de Trichogramma pretiosum. Iheringia, Série Zoologia, 107:e2017015.

Vargas, R. I.; Piñero, J. C.; Leblanc, L.; Manoukis, N. C. \& MaU, R. F. L. 2016. Area wild management of fruit flies (Diptera: Tephritidae) in Hawaii. In: Ekesi, S.; Mohamed, S. A. \& DE Meyer, M. eds. Fruit fly research and development in Africa - towards a sustainable management strategy to improve horticulture. Switzerland, Springer, p. 673-694.

Vet, L. E. M.; Lewis, W. J. \& CARDÈ, R. T. 1995. Parasitoid foraging and learning. In: CARDÈ, R. T. \& Bell, W. J. eds. Chemical Ecology of Insects. London, Chapman and Hall, p. 65-101.

Villagra, C. A.; Pennacchio, F. \& Niemeyer, H. M. 2007. The effect of larval and early adult experience on behavioural plasticity of the aphid parasitoid Aphidius ervi (Hymenoptera, Braconidae, Aphidiinae). Naturwissenschaften 94:903-910.

VINSON, S. B. 1998. The general host selection behavior of parasitoid Hymenoptera and a comparison of initial strategies utilized by larvaphagous and oophagous species. Biological Control 11:79-96.

WARdLe, A. R. \& Borden, J. H. 1985. Age-dependent associative learning by Exeristes roborator (F.) (Hymenoptera: Ichneumonidae). The Canadian Entomologist 117:605-616.

Wyckhuys, K. A. G. \& Heimpel, G. E. 2007. Response of the soybean aphid parasitoid Binodoxys communis to olfactory cues from target and non-target host-plant complexes. Entomologia Experimentalis et Applicata 132:149-158.

Zhang, S.; Chen, L. Z.; Gu, S. H.; CuI, J. J.; GAO, X. W.; Zhang, Y. J. \& GuO, Y. Y. 2011. Binding Characterization of Recombinant Odorantbinding Proteins from the Parasitic Wasp, Microplitis mediator (Hymenoptera: Braconidae). Journal of Chemical Ecology 37:189-194.

Zhou, J. J. 2010. Odorant-Binding Proteins in Insects. In: LITWACK, G. eds. Vitamins and Hormones. Burlington, Academic Press, p. 241-272. 